\title{
Effect of Egg Weight on External and Internal Qualities, Physiological and Hatching Success of Japanese Quail Eggs (Coturnix coturnix japonica)
}

http://dx.doi.org/10.1590/1806-9061-2018-0777

EAuthor(s)

Hegab IMI,I,III (iD https://orcid.org/0000-0001-7430-3600

Hanafy AM (D) https://orcid.org/0000-0002-8303-9896

College of Grassland Science, Gansu Agricultural University, Lanzhou 730070, China.

" Gansu Agricultural University-Massey University Research Centre for Grassland Biodiversity, Gansu Agricultural University, Lanzhou 730070, China.

III Department of Hygiene, Zoonosis and Animal Behavior and Management, Faculty of Veterinary Medicine, Suez Canal University, Ismailia, 41522, Egypt.

Iv Department of Animal Production, Faculty of Agriculture, Suez Canal University, 41522 Ismailia, Egypt.

\section{-Mail Address}

Corresponding author e-mail address Dr.Ahmed M. Hanafy

Department of Animal Production, Faculty of Agriculture, Suez Canal University, 41522

Ismailia, Egypt.

Phone: 002-01224784599

Email: ahmed_hanafy@agr.suez.edu.eg

\section{-Keywords}

Egg size; egg quality; hatchability; hormones; parameters.

\section{ABSTRACT}

This experiment was conducted to evaluate the effect of egg weight and egg physical characteristics on embryonic development, hatchability, and hatchling weight of Japanese quails (Coturnix coturnix japonica). In total, 689 eggs were classified in two categories: small $(<13.5 \mathrm{~g})$ or large $(\geq 13.5 \mathrm{~g})$, and different external eggshell and internal quality traits were measured. On days 6 and 14 of incubation, tissue triiodothyronine (T3) and thyroxine (T4) were extracted from embryos in both egg sizes and analyzed. Relative to internal egg-quality traits, large eggs had significantly higher yolk height, yolk diameter, yolk weight, albumen height, and albumen weight than small eggs $(p \leq 0.01)$. However, Haugh unit score $(p=0.27)$ was not significantly different between the two egg sizes. Relative to eggshell quality parameters, large eggs had significantly higher total pore count, surface area, eggshell volume, and eggshell weight than small eggs ( $p \leq 0.05)$, with consequent higher hatchability rate and hatchling weight. Pearson's correlation coefficients revealed significant correlations $(p \leq 0.05)$ between egg weight and different external and internal egg quality parameters. Thyroid hormone levels were not significantly different between egg groups on d 6 day, while on d 14, a significant difference was recorded ( $p \leq 0.05)$. In conclusion, larger egg sizes are recommended to obtain better hatchability, lower embryonic death rates, and heavier hatchlings compared with smaller eggs of Japanese quails.

\section{INTRODUCTION}

The physical properties of quail eggs play a critical role on embryo development and influence hatchability. These parameters have the potential to meet the demands of fast-growing quail embryos (Bai et al., 2016) regarding nutrition, physical protection, and subsequent smooth hatching procedure to produce viable chicks. Both egg external quality parameters, such as egg weight, eggshell traits, egg surface area and egg volume (Boleli et al., 2016), and internal traits, such as yolk and albumen height and weight (Demirel \& Kirikci, 2009), may directly or indirectly influence hatchability results. For example, egg weight affects both hatchability and hatchling weight (Iqbal et al., 2016). Similarly, internal egg quality traits, such as yolk and albumen contents, influence embryo development and hatchling livability (Sahan et al., 2014).

The main functions of the eggshell are protection and supply of calcium and other mineral to the embryos and to allow gas and water exchange between the embryos and the external environment, which is essential for the success of the entire incubation and hatching processes (Portugal et al., 2014). Specifically, gas exchange occurs by diffusion through microscopic pores on the surface of the eggshell (Rahn et al., 1987), and hence, depends on two main key factors: pore counts and eggshell thickness (Rahn et al., 1974). Both traits also modify eggshell 
water-exchange properties by changing egg weight loss percentage (EWL\%) during incubation. Both lower pore numbers-high eggshell thickness or higher pore count-low eggshell thickness impair gas exchange, ultimately causing embryonic mortality (Soliman et al., 1994).

Most studies investigating the influence of egg internal quality parameters on embryonic development and hatchability traits (Khan et al., 2013; Zita et al., 2012) have focused on albumen and yolk quality. In addition of supplying essential nutrients for embryo growth and development, the albumen also protects embryos from exposure to pathogenic microorganisms (Yuan et al., 2013), and hatchability rates have been linked to changes in albumen quality (Demirel \& Kirikci, 2009). Yolk morphometric measurements can be obtained by mathematical calculations and yolk amount and composition are known to impact of post-hatching yolk sac nutrient reservoirs (UlmerFranco et al., 2010). Several major (egg storage time and conditions, and hen strain and age) and minor (nutrition in specific cases) factors have been connected to changes in internal egg quality traits (Osei-Amponsah et al., 2014).

Although there is a considerable body of literature devoted to the analysis of egg internal and external quality traits, fewer studies have focused on common factors that may govern both traits. One of the leading factors that may alter or shape both traits in poultry - unquestionably together with other factors - is egg weight or egg size (Onbasilar et al., 2011). Egg size influences eggshell characteristics and egg external quality traits and it is well accepted that egg size influences incubation success, embryonic death, and hatchability \% (Shafey, 2002). Moreover, yolk volume is lower in smaller than in larger eggs, and consistently increases with egg size; therefore, the solid contents of large eggs may be significantly different than those of small ones. These variations may be explained by differences in egg weight, which modifies the relative yolk and albumen masses.

Egg quality is a general term referring to several standards which define both internal and external quality. External egg quality is focused on eggshell cleanliness, texture, and shape, where as internal quality refers to egg white (albumen) clarity and viscosity, air cell size, yolk shape, and yolk membrane strength. In addition of their functional importance, these parameters are typically used to determine egg quality from several points of view, including egg nutritional content, egg integrity for commercialization, storage and incubation, as well as preservation during storage. The yolk and the albumen are the main organic components of the egg, supplying 32 and $1 \%$ of fat and 16 and $11 \%$ of protein, respectively (Willems et al., 2014). In addition, the modern egg industry requires specific egg integrity criteria for proper commercialization, such as eggshell integrity and color, which significantly influence the visual perception of the consumers, and consequently its market acceptance (Maxkwee et al., 2014). During storage and incubation, eggshell strength and integrity are important for preventing water loss, microbial penetration, and untimely gas diffusion prior to incubation (Cook et al., 2015). Eggshell integrity, besides the soundness of the internal constituents, markedly affect embryo viability during egg storage, when changes in the embryo or in certain egg physical aspects, such as albumen $\mathrm{pH}$, causes movement of nutrients from the albumen to the blastoderm, and may reduce resistance to gas diffusion, causing necrosis and regressive changes in the blastoderm, which result in embryo development failure (Egbeyale et al., 2013). Therefore, every effort should be devoted to ensuring optimal egg internal and external quality for storage and incubation.

Thyroid hormones are essential for the correct development of nearly every structure of the embryo body from the very early stages of development. For example, thyroid hormones are crucial endocrine regulators of muscular development of the embryos, hatching process, and post-hatch metabolism (Too et al., 2017). Plasma T3 level significantly increases when the embryo starts lung respiration at the late embryonic stage (Christensen \& Davis, 2004), and embryo survival rates improve when the level of embryonic thyroid hormones increase. Indeed, there is limited information about the correlation between egg weight and the external and internal egg quality traits in Japanese quail (Hanafy \& Hegab, 2019), and, to the best of our knowledge, no studies have yet been published on the relation of embryonic thyroid hormone levels and egg weight. Therefore, the objective of this study was to compare the physical characteristics of light and heavy Japanese quail eggs and to evaluate the effects of possible relations among these traits on thyroid hormone profiles, hatchability, and hatchling weight.

\section{MATERIALS AND METHODS}

\section{Birds and egg incubation}

The study was conducted at the Department of Animal Production, Faculty of Agriculture, Suez Canal University, Ismailia, Egypt. A total of 689 Japanese 
quail eggs was obtained from a commercial breeding flock (18-20 weeks old) with a sex ratio of 2 females: 1 male. All eggs were collected (within three days), stored in an egg-storage room at $18^{\circ} \mathrm{C}$ and $75 \%$ relative humidity, and identified. Eggs were weighed immediately before incubation $\left(\mathrm{W}_{0}\right)$ on an electronic balance (0.01-gaccuracy), and then classified as small $(12.23 \pm 0.04 \mathrm{~g})$ or large $(14.32 \pm 0.04 \mathrm{~g})$. Out of this total, 238 eggs [small/n=125 (12.62 \pm 0.06$)$, large $/ n=113(14.84 \pm 0.06)]$ were incubated and the remaining 451 eggs [small/ $n=278(11.83 \pm 0.05)$, large/ $n=173(13.80 \pm 0.07)]$ were opened to evaluate their internal parameters.

The incubation conditions (C3 PTO single stage incubator, Alexandria, Egypt) were $37.5^{\circ} \mathrm{C}$ and $65 \%$ relative humidity. The incubated eggs were turned 12 times per day (every two hours) in a $45^{\circ}$ angle. On d 15 of incubation, eggs were weighed again $\left(W_{F}\right)$. Hatchlings were weighed at hatch.

On d 18 of incubation, unhatched eggs were opened and inspected for embryonic development stage (EDS). Clear eggs that did not show any embryonic development were considered infertile. Eggs were classified as infertile, early-dead embryos (died before d 5 of incubation), late dead embryos (d 5 to pipping), pipped, and hatched (Egg type). Hatchability rate was calculated by dividing the number of hatched chicks by the number of fertile eggs set.

\section{Measurement of external egg quality traits}

Before setting, eggs were individually weighed on a digital balance (0.01-g accuracy) in the beginning of the experiment $\left(W_{0}\right)$. Egg length and breadth were measured using digital calipers (Mitutoyo, Mizonokuchi, Japan) to the nearest $0.01 \mathrm{~mm}$, and egg shape index was calculated as breadth to length ratio $\times 100$. Surface area $(S)$ was calculated as $S=4.835 \times$ $W^{0.662}$, where $W=$ egg weight (Paganelli et al., 1974). Egg volume $(V)$ was calculated as $V=(0.6057-0.0018 B)$ $L B^{2}$, where: $L$ is egg length and $B$ is maximum egg breadth.

At the end of the incubation period, four eggshell regions were selected from each eggshell: one from the large end, two from the equator, and one from the pointed end. Eggshell processing and staining were conducted according to Soliman et al. (1994). Pores of all incubated eggs were directly counted under a dissecting microscope (2.5x magnification) and expressed as number of pores $/ 0.25 \mathrm{~cm}^{2}$. Total pore count was calculated per egg by multiplying eggshell surface area $\times$ (average pore count/egg $\times 4)$. Eggshell thickness ( $\mu \mathrm{m})$ without the eggshell membrane was evaluated at the four eggshell regions using a micrometer. Egg weight loss rate (EWL\%) was calculated as EWL\% $=\left[\left(W_{0}-W_{F}\right) / W_{0}\right] \times 100$, where $W_{0}$ is egg weight at setting and $W_{F}$ is egg weight at transfer to the hatcher on d 15 of incubation.

\section{Measurement of internal egg quality traits}

Yolk diameter and weight were measured in $\mathrm{mm}$ using a Vernier caliper and an electronic balance (0.01-g accuracy), respectively. Albumen and yolk heights were measured at their highest point using a spherometer. After removal of the egg contents, the eggshell was weighed (g) using an electronic balance. Albumen weight (g) was calculated as egg weight (yolk weight + eggshell weight). Haugh units ( $\mathrm{HU})$ were calculated as $\mathrm{HU}=100 \times \log$ (albumen height $-1.7 \times$ egg weight $\left.{ }^{0.37}+7.6\right)$, according to Fernandez et al. (2011).

\section{Extraction and quantification of triiodo- thyronine (T3) and thyroxine (T4) in embry- onic tissues}

On days 6 and 14 of incubation, T3 and T4 hormones were extracted from tissue samples of 10 embryos per egg size: 5 on d 6 (whole embryo) and 5 on d 14 (breast muscle), totaling 20 embryos. Samples were collected, flash-frozen in liquid nitrogen, and then preserved at $-80{ }^{\circ} \mathrm{C}$ until further processing. After thawing, tissue samples were then homogenized in an ultrasound sonicator (Model Q55, QSONICA, Newtown, USA) at a ratio of $1 \mathrm{mg}$ tissue/ $1 \mathrm{~mL}$ phosphate buffer saline (PBS). After homogenization, samples were centrifuged for $15 \mathrm{~min}$ at $4000 \mathrm{rpm}$. The supernatant was collected and analyzed for T3 and T4 using commercial ELISA kits (BioCheck ${ }^{\circledR}$, Foster City, USA), according to the manufacturer's instructions.

\section{Statistical analysis}

The SPSS 22.00 software was used for all analyses. Independent t-test was performed to test the significance of egg parameter and thyroid hormonal profile differences between small and large eggs. When data set failed to meet the assumption of normality of distribution, Mann-Whitney $U$ test was used. Twoway analysis of variance (ANOVA) was applied, with egg size and stage of embryonic development as fixed variables, and pore count, eggshell thickness, and EWL\% as dependent variables. Duncan's post hoc multiple comparisons test was conducted where 
appropriate. Pearson's correlation coefficient was used to assess the strength of association between egg size and different internal and external egg quality traits. The level of significance at which the null hypothesis was rejected was $\alpha=0.05$.

\section{RESULTS}

Table 1 shows that large eggs had significantly higher $(p<0.05)$ weight, hatchability rate, hatchling weight, total pore count, surface area, and eggshell volume than small eggs. On the other hand, small eggs lost significantly more $(p<0.05)$ weight during incubation than large eggs, while fertility rate, average pore count/0.25 $\mathrm{cm}^{2}$, eggshell thickness, and egg shape index values were not significantly $(p>0.05)$ different between egg sizes.

The two-way ANOVA revealed that neither egg size $(F=0.59, p=0.44)$ nor size $\times E D S$ interaction $(F=0.59$, $p=0.67$ ) had any significant effect on pore count; however, pore count was significantly different among EDS classes ( $F=3.30, p=0.01)$, and Duncan's post-

Table 1 - Functional egg quality traits of small and large eggs of Japanese quails.

\begin{tabular}{lccc}
\hline Parameter & Small eggs & Large eggs & $p$-value \\
\hline Egg weight (g) & $12.62 \pm 0.06$ & $14.84 \pm 0.06$ & $\mathrm{t}=22.65, p<0.0001$ \\
Fertility (\%) & 83.20 & 80.53 & $X=0.29, P=0.62$ \\
Hatchability (\%) & 67.42 & 78.64 & $X=141.69, p<0.0001$ \\
Chick weight $(\mathrm{g})$ & $7.95 \pm 0.29$ & $9.12 \pm 0.32$ & $U=589.50, p<0.0001$ \\
EWL $(\%)$ & $16.08 \pm 1.33$ & $12.36 \pm 0.78$ & $U=6023.00, p=0.03$ \\
Pore count/0.25(m² & $34.16 \pm 0.93$ & $32.62 \pm 0.89$ & $U=3309.00, p=0.36$ \\
Total pore count/egg & $340.94 \pm 7.97$ & $370.40 \pm 9.80$ & $\mathrm{t}=2.35, P=0.02$ \\
Eggshell thickness $(\mathrm{mm})$ & $0.20 \pm 0.002$ & $0.20 \pm 0.002$ & $U=3590.00, p=0.98$ \\
Surface area $\left(\mathrm{cm}^{2}\right)$ & $2.59 \pm 0.08$ & $2.84 \pm 0.08$ & $\mathrm{t}=22.11 p<0.0001$ \\
Egg volume $\left(\mathrm{cm}^{3}\right)$ & $13.27 \pm 0.08$ & $14.74 \pm 0.07$ & $\mathrm{t}=13.54, p<0.0001$ \\
Shape index\% & $78.55 \pm 0.37$ & $78.10 \pm 0.25$ & $U=6824.00, p=0.65$ \\
\hline
\end{tabular}

$p$-values $\leq 0.05$ indicate values in the same row are statistically different. $t=$ independent samples $t$-test, $X=$ Chi-square test, $U=$ Mann-Whitney $U$ test.

hoc test showed the highest pore concentration was found in hatched eggs (supplemental Table 1). Egg size did not significantly influence eggshell thickness $(F=0.18, p=0.67)$, which, however, was significantly influenced by EDS class ( $F=8.29, p=0.01)$ and size $\times$ EDS class interaction $(F=4.58, p=0.01)$. Duncan's post-hoc multiple comparison test revealed that the thickest eggshells were obtained in infertile and earlydead eggs (supplementary Table 1). Finally, egg size $(F=5.00, p=0.03)$, EDS class $(F=17.90, p=0.01)$ and size

Supplementary Table 1 - Two-way analysis of variance (ANOVA) between egg size and different types of eggs of Japanese quails as fixed variables with pore count, eggshell thickness, egg weight loss (\%) as dependent variables.

\begin{tabular}{lcc}
\hline$p$-value & F-value & Source of variance \\
\hline 0.44 & 0.59 & Pore count \\
0.01 & 3.30 & Egg size \\
0.67 & 0.59 & Egg type \\
\hline & & Egg size $\times$ type interaction \\
\hline 0.67 & 0.18 & Eggshellthickness \\
$<0.0001$ & 8.29 & Egg size \\
$<0.0001$ & 4.58 & Egg size $\times$ type interaction \\
\hline & & Egg weight loss \\
\hline 0.03 & 5.00 & Egg size \\
$<0.0001$ & 17.90 & Egg type \\
0.02 & 3.10 & Egg size $\times$ type interaction \\
\hline
\end{tabular}

$\times$ EDS class interaction ( $F=3.10, p=0.02$ ) significantly influenced EWL\% during the incubation period. Duncan's post-hoc tests comparing egg EDS classes determined the highest EWL\% in early-dead eggs, while the egg size $\times$ EDS class interaction detected the lowest EWL\% $(9.52 \pm 0.50)$ in hatched large-sized eggs amongst all size $\times$ type groups interactions (supplementary Table 1).

Large eggs presented significantly greater yolk height, yolk diameter, yolk weight, albumen height, albumen weight and eggshell weight values than small eggs (Table 3). However, Haugh unit score $(p=0.27)$ did not significantly differ between egg sizes. The correlations between egg weight and some egg quality traits are presented in Table 4. Egg weight was positively correlated with eggshell weight, yolk weight, yolk diameter, albumen weight, hatchling weight, and egg volume, and negatively correlated with pore count and EWL\%. The correlation between egg weight and eggshell thickness $(r=0.11)$ was low and not significant $(p=0.18)$, as well as with yolk and albumen heights ( $p=0.17$ and $p=0.26$, respectively).

Figure 1 shows that neither T3 nor T4 levels in embryo tissues on $d 6$ of incubation were significantly different between egg sizes ( $T 3 ; t=-1.20, p=0.26$, T4; $t=0.58, p=0.58)$. However, large eggs showed 
Table 2 - Pore count, eggshell thickness, and egg weight loss (EWL\%) during of different types of Japanese quail eggs.

\begin{tabular}{lccc}
\hline Egg type & Pore count/ $0.25 \mathrm{~cm}^{2}$ & Eggshellthickness $(\mathrm{mm})$ & \multicolumn{1}{c}{ EWL\% } \\
\hline Infertile & $30.60 \pm 1.44^{\mathrm{b}}$ & $20.91 \pm 0.30^{\mathrm{a}}$ & $13.17 \pm 1.04^{\mathrm{b}}$ \\
Early dead & $30.87 \pm 1.58^{\mathrm{ab}}$ & $20.86 \pm 0.40^{\mathrm{a}}$ & $23.33 \pm 1.43^{\mathrm{a}}$ \\
Late dead & $30.56 \pm 1.81^{\mathrm{b}}$ & $19.88 \pm 0.35^{\mathrm{b}}$ & $14.33 \pm 1.38^{\mathrm{b}}$ \\
Pipped & $29.43 \pm 3.59^{\mathrm{b}}$ & $18.77 \pm 0.73^{\mathrm{b}}$ & $12.09 \pm 3.42^{\mathrm{bc}}$ \\
Hatched & $34.92 \pm 0.78^{\mathrm{a}}$ & $19.23 \pm 0.18^{\mathrm{b}}$ & $10.27 \pm 0.64^{\mathrm{c}}$ \\
Level of significance & $\mathrm{F}=3.30, P=0.01$ & $\mathrm{~F}=8.29, P=0.01$ & $\mathrm{~F}=5.00, P=0.03$ \\
\hline
\end{tabular}

Means within the same column with different superscripts are significantly different at $p \leq 0.05$.

Table 3 - Effect of egg size on different internal egg quality parameters and on eggshell weight in Japanese quails.

\begin{tabular}{lccc}
\hline Parameter & Small egg & Large egg & $p$ value \\
\hline Egg weight $(\mathrm{g})$ & $11.83 \pm 0.05$ & $13.8 \pm 0.05$ & $\mathrm{t}=-27.19, p<0.0001$ \\
Yolk height/mm & $10.34 \pm 0.09$ & $10.93 \pm 0.09$ & $\mathrm{t}=-4.35, p<0.0001$ \\
Yolk diameter/mm & $26.36 \pm 0.11$ & $27.42 \pm 0.13$ & $\mathrm{t}=-6.20, p<0.0001$ \\
Yolk weight/g & $3.61 \pm 0.06$ & $4.28 \pm 0.03$ & $\mathrm{t}=-8.51, p<0.0001$ \\
Albumen height/mm & $4.53 \pm 0.07$ & $4.87 \pm 0.09$ & $\mathrm{t}=-3.10, p<0.0001$ \\
Albumen weight/g & $6.98 \pm 0.05$ & $8.39 \pm 0.08$ & $\mathrm{t}=-15.62, p<0.0001$ \\
Eggshell weight/g & $1.07 \pm 0.02$ & $1.23 \pm 0.02$ & $\mathrm{t}=-8.60, p<0.0001$ \\
Haugh unit score & $89.57 \pm 0.34$ & $90.20 \pm 0.45$ & $\mathrm{t}=-1.11, p=0.27$ \\
\hline
\end{tabular}

$p$-values $\leq 0.05$ indicate values in the same row are statistically different. $t=$ independent samples $t$-test.

Table 4 - Correlations between egg weight and some egg quality traits

\begin{tabular}{lccccccccccc}
\hline Trait & $\begin{array}{c}\text { Eggshell } \\
\text { weight }\end{array}$ & $\begin{array}{c}\text { Yolk } \\
\text { height }\end{array}$ & $\begin{array}{c}\text { Yolk } \\
\text { diameter }\end{array}$ & $\begin{array}{c}\text { Yolk } \\
\text { weight }\end{array}$ & $\begin{array}{c}\text { Albumen } \\
\text { height }\end{array}$ & $\begin{array}{c}\text { Albumen } \\
\text { weight }\end{array}$ & $\begin{array}{c}\text { Pore } \\
\text { count }\end{array}$ & $\begin{array}{c}\text { Shell } \\
\text { thickness }\end{array}$ & $\begin{array}{c}\text { Chick } \\
\text { weight }\end{array}$ & $\begin{array}{c}\text { EWL } \\
\text { volume }\end{array}$ \\
\hline $\begin{array}{l}\text { Egg weight } \\
\begin{array}{l}\text { Correlation } \\
\text { coefficient }\end{array}\end{array}$ & $0.12^{*}$ & $0.08^{\text {Ns }}$ & $0.35^{* * *}$ & $0.13^{* *}$ & $0.07^{\text {Ns }}$ & $0.82^{* * *}$ & $-0.18^{* *}$ & $0.11^{\text {Ns }}$ & $0.35^{* *}$ & $-0.13^{* *}$ & $0.87^{* * *}$ \\
$p$ value & 0.01 & 0.17 & $\leq 0.0001$ & 0.01 & 0.26 & $\leq 0.01$ & 0.02 & 0.18 & $\leq 0.0001$ & 0.04 & $\leq 0.0001$ \\
\hline
\end{tabular}

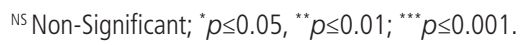

significantly higher T3 levels $(t=-2.54, p=0.04)$ and significantly lower T4 levels $(t=2.79, p=0.02)$ on $d 14$ of incubation compared to small eggs.

\section{DISCUSSION}

Our results clearly demonstrate that both external and internal egg quality traits are different between Japanese quail egg weight categories and influence their hatchability rate. Table 1 shows that, although no fertility differences were detected between egg sizes, significantly higher hatchability was observed in large eggs compared with small eggs. These results are consistent with Alabi et al. (2012a), who reported higher hatchability in large than in medium and small eggs of an indigenous South African chicken breed. However, other studies demonstrated that mediumsized eggs have better hatchability compared with small and large eggs in broilers (Abiola et al., 2008). This discrepancy among literature results may arise from differences in egg weight classification, as well as in poultry species and breeds (Alabi et al., 2012b). Also, the significant positive correlation detected between egg and hatchling weights, with heavier hatchlings obtained from larger eggs, is consistent with the study of lqbal et al. (2016) with broilers.

Although average pore count $/ 0.25 \mathrm{~cm}^{2}$ and eggshell thickness were similar between egg sizes (Table 1), small eggs lost significantly more weight during incubation than large eggs. These findings are in agreement with Abiola et al. (2008), who recorded the lowest egg weight loss in large eggs and the highest in small eggs. Large eggs have a greater amount of albumen (and thus greater moisture content) than small eggs. According to Ulmer-Franco et al. (2010), as the albumen has high water holding capacity, the higher the albumen contents, the higher the water-keeping capacity of the egg. Moreover, small eggs have a relatively higher volume/surface ratio (5.12) compared with large eggs (5.19), which ultimately leads to higher egg weight loss (Iqbal et al., 2016). The higher egg weight loss determined in small eggs in the present study may also explain their lower hatchability. If water loss is too high, embryonic growth is compromised, and, consequently, hatchability will decrease. Our results also clearly show a significant negative correlation between egg weight and egg weight loss (Table 4), which is consistent with the findings of Abanikannda 
et al. (2011), and may indicate slower weight loss in larger eggs than in relatively smaller eggs. Moreover, in the present study, the correlation between egg weight and eggshell thickness was not significant (Table 4), in agreement with the reports of Elamin et al. (2014) in broiler breeders. Also, a significant negative correlation between egg weight and average pore count $/ 0.25 \mathrm{~cm}^{2}$ (Table 4) was found, with an increase in egg size corresponding to a decrease in average pore count $/ 0.25 \mathrm{~cm}^{2}$. However, independently of average pore count $/ 0.25 \mathrm{~cm}^{2}$, total pore count per egg was significantly higher in large eggs than in small ones, in agreement with the results obtained by Hegab et al. (2008), who showed that larger eggs have higher pore counts than smaller eggs.

The results in Table 2 show that average pore count/ $\mathrm{cm}^{2}$, eggshell thickness, and egg weight loss percentage significantly influenced embryonic mortality rates. The highest EWL \% was found in early-dead eggs. This may be due to some functional alterations, such as poor albumen quality and/or other physical egg attribute, as pore count and eggshell thickness (Table 2) did not show any remarkable results (Soliman et al., 1994). On the other hand, Saylam \& Sarica (1999) reported the highest egg weight loss in hatched (29.26\%) Japanese quail eggs compared with infertile eggs and those with early and late embryo mortality. This disagreement may be attributed to the incubation conditions, breeding season, and/or flock age, as egg porosity and internal egg quality differences have been reported among different breeder ages (Massaro \& Davis, 2004), ultimately leading to excessive weight loss. Hatched eggs presented the highest number of pores (Table 2 ), which may have allowed better regulation of gas and water vapor exchange between the embryo and the external environment. Saylam \& Sarica (1999) also observed higher number of pores in hatched eggs. On the other hand, it is recognized that the embryo mobilizes the calcium required for hard tissues and bone development during embryonic development from two extra-embryonic sources: first from the yolk, and then from the eggshell (Tuan and Ono, 1986). Therefore, over the course of incubation, embryos in late dead, pipped, and hatched eggs mobilize more calcium from the eggshell, resulting in thinner eggshells. Our results confirmed this assumption, as the thickest eggshell was measured in infertile and early dead embryos compared with late dead, pipped, and hatched eggs (Table 2).

Higher values of all internal egg quality parameters were determined in large eggs (Table 3 ) than in small eggs, and egg weight was positively correlated with eggshell weight, yolk diameter, and yolk weight (Table 4). Albumen is a chief marker of internal egg quality parameters and the main reservoir of water and protein, regulating water exchange between the yolk and the developing embryos (Willems et al., 2014). Khan et al. (2014) suggested that embryonic mortality increases may be due to water loss and albumen degradation. Indeed, extended water loss results in albumen degradation that, in turn, causes blastoderm to move near the eggshell, resulting in early embryonic death (Brake et al., 1993). Such changes in specific physical properties of the egg, specifically albumen $\mathrm{pH}$, causes the movement of nutrients from the albumen to the blastoderm and may decrease the resistance to gas flow, which can damage embryonic development (Lapao et al., 1999). Also, the egg yolk is a vital resource of nutrients and the primary storage site of trace minerals for the embryos and hatchlings. This supports the concept that larger eggs result larger chicks due to the better nutritional conditions of the embryos during incubation. Although Haugh unit is an objective mathematical indicator of albumen quality, it is usually used to measure the degree of albumen deterioration during prolonged egg storage periods (Zita et al., 2012). In our study, both egg weight classes were subjected to equal storage periods, and therefore, the assumption is that both egg classes will possess nearly equal Haugh units.
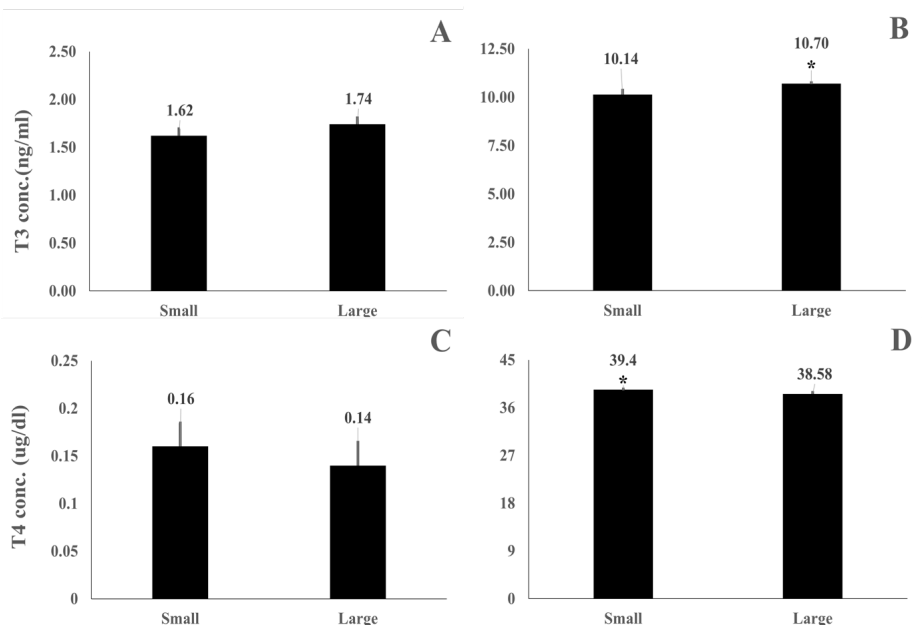

Figure 1 - Mean concentrations of T3 [days 6 (A) and 14 (B) of incubation] and T4 [days 6 (C) and 14 (D) of incubation] in small and large eggs. * Significant at $p \leq 0.05$ with independent samples t-test.

Figure 1 shows that egg weight did not significantly affect ( $p>0.05$ ) T3 and T4 tissue levels on $d 6$ of incubation, indicating that these were of maternal origin. However, on d 14 of incubation, there was a significant effect of egg weight on these parameters, with higher T3 levels determined in large eggs than in small eggs, while T4 levels presented the opposite 
behavior. Higher thyroid hormone levels have been linked to better hatchability in different avian species, including Japanese quails (Khalil, 2009). Thyroid hormones are known to play crucial roles in the metabolic rate, differentiation and maturation of avian embryos throughout the incubation period via different mechanisms. According to Decuypere et al. (2005), they regulate the basal metabolic rate and maintain constant body temperature, as well as influence protein and lipid metabolism and control the growth of embryos. During the initial stages of incubation, low thyroid hormone levels have anabolic effects, promoting better embryonic growth, while the higher levels observed in the later stages limit embryo growth. This bi-modal effect is deployed for better growth and hatchability traits.

In conclusion, our study suggests that egg size significantly influences internal and external egg quality traits, as well as embryo thyroid hormone profile. These functional components of egg quality traits are essential, under standard incubation conditions, to increase the hatchability of incubated eggs and hatchling weight. Therefore, larger egg sizes are recommended to obtain better hatchability, lower embryonic death rates, and heavier hatchlings compared with smaller eggs of Japanese quails.

\section{AUTHOR CONTRIBUTION}

The authors equally contributed to perform experimental procedures and data analyses, and to draft this manuscript.

\section{REFERENCES}

Abanikannda O, Leigh A, Giwa A. Influence of egg weight, breed and age of hens on weight loss of hatching broiler eggs. Archiva Zootechnica 2011;14:343-356

Abiola S, Meshioye O, Yerinde O, Bamglose MA. Effect of egg size on hatchability of broiler chicks. Archiva Zootechnica 2008;57:83-86.

Alabi J, Ng'ambi W, Norris D. Effect of egg weight on physical egg parameters and hatchability of indigenous Venda chickens. Asian Journal of Animal and Veterinary Advances 2012a;7:166-172.

Alabi O, Ng'ambi J, Norris D, Mabelebele M. Effect of egg weight on hatchability and subsequent performance of Potchefstroom Koekoek. Asian Journal of Animal and Veterinary Advances 2012b;7:718-725.

Bai J, Pang Y, Zhang X, Li Y. Study on the morphological development of quail embryos. Revista Brasileira de Ciência Avícola 2016;18:91-93.

Boleli I, Morita V, Matos Jr J, Thimotheo M, Almeida V. Poultry egg incubation: integrating and optimizing production efficiency. Revista Brasileira de Ciência Avícola 2016;18:1-16.

Brake J, Walsh T, Vick S. Hatchability of broiler eggs as influenced by storage and internal quality. Zootecnica International 1993;16:30-41.
Christensen VL, Davis GS. Maternal dietary iodide influences turkey embryo thyroid function. Poultry Science 2004;3:550-557.

Cook MI, Beissinger SR, Toranzos GA, Arendt WJ. Incubation reduces microbial growth on eggshells and the opportunity for trans-eggshell infection. Ecology Letters 2005;8:532-537.

Decuypere E, Van As P, Van der Geyten S, Darras VM. Thyroid hormone availability and activity in avian species: a review. Domestic Animal Endocrinology 2005;29:63-77.

Demirel S, Kirikci K. Effect of different egg storage times on some egg quality characteristics and hatchability of pheasants (Phasianus colchicus). Poultry Science 2009;88:440-444.

Egbeyale LT, Bosa MK, Sogunle OM, Adeleye OO. Effect of preincubation storage periods on weight loss, embryonic development, and hatchability of pullet eggs. The Pacific Journal of Science and Technology, 2013;14:416-424.

Elamin km, Malik HEE, Sakin AIY, Elagib HAA, Dousa BM. Effect of egg weight and egg eggshell thickness on hatchability and embryonic mortality of cobb broiler breeder eggs. Global Journal of Animal Scientific Research 2015;3(1).

Fernandez, IB, Cruz, VC , Polycarpo, GV. Effect of dietary organic selenium and zinc on the internal egg quality of quail eggs for different periods and under different temperatures. Brazilian Journal of Poultry Science $2011 ; 13(1): 35-41$

Hanafy, AM, Hegab IM. Effects of egg weight and light sources during incubation period on embryonic development and post-hatch growth of Japanese quail (Coturnix japonica). European Poultry Science $2019 ; 83$.

Hegab IM, Mohammad M, Fares I, Maha MT, Ashraf MMK. Suitability of ostrich eggs to hatchability. Suez Canal Veterinary Medicine Journal 2008;13:367-375

Iqbal J, Khan SH, Mukhtar N, Ahmed T, Pasha RA. Effects of egg size (weight) and age on hatching performance and chick quality of broiler breeder. Journal of Applied Animal Research 2016;44:54-64.

Khalil H. Productive and physiological responses of Japanese quail embryos to light regime during incubation period. Slovak Journal of Animal Science 2009;42:79-86.

Khan MJA, Khan S, Amin M, Bukhsh A. The effect of storage time on egg quality and hatchability characteristics of Rhode Island Red (RIR) hens. Veterinarski Arhive 2014;84:291-303.

Khan MJA, Khan SH, Bukhsh A, Abbass MI, Javed M. Effect of different storage period on egg weight, internal egg quality and hatchability characteristics of fayumi eggs. Italian Journal of Animal Science 2013;12:e51.

Lapao C, Gama LT, Soares MC. Effects of broiler breeder age and length of egg storage on albumen characteristics and hatchability. Poultry Science 1999;78:640-5.

Massaro M, Davis LS. The influence of laying date and maternal age on eggshell thickness and pore density in yellow-eyed penguins. The Condor 2004;106:496-505

Maxkwee EN, Perry JJ, Lee K. Flavor and appearance of whole eggshell eggs made safe with ozone pasteurization. Food Science \& Nutrition 2014;2:578-84

Onbasilar EE, Erdem E, Poyraz O, Yalcin S. Effects of hen production cycle and egg weight on egg quality and composition, hatchability, duckling quality, and first-week body weight in Pekin ducks. Poultry Science 2011;90:2642-2647. 
Osei-Amponsah R, Kayang BB, Manu H, Naazie A. Egg quality traits of local Ghanaian chickens and influence of storage period. Animal Genetic Resources 2014;55:93-99.

Paganelli CV, Olszowka A, Ar A. The Avian egg: surface area, volume, and density. The Condor 1974;76:319-325.

Portugal SJ, Maurer G, Thomas GH, Hauber ME, Grim T, Cassey P. Nesting behaviour influences species-specific gas exchange across avian eggshells. Journal of Experimental Biology 2014;217:3326-3332.

Rahn H, Paganelli CV, Ar A. Pores and gas exchange of avian eggs:a review. The Journal of Experimental Zoology 1987;1(Supl):165-172.

Rahn H, Paganelli CV, Ar A. The avian egg:air-cell gas tension, metabolism and incubation time. Respiration Physiology 1974;22:297-309.

Sahan U, Ipek A, Sozcu A. Yolk sac fatty acid composition, yolk absorption, embryo development, and chick quality during incubation in eggs from young and old broiler breeders. Poultry Science 2014;93:2069-2077.

Saylam S, Musa S. Effects of eggshell thickness, eggshell pores and egg weight loss on hatchability on Japanese quail eggs. Turkish Journal of Veterinary and Animal Sciences 1999;23:41-46.

Shafey TM. Eggshell conductance, embryonic growth, hatchability and embryonic mortality of broiler breeder eggs dipped into ascorbic acid solution. British Poultry Science 2002;43:135-140.
Soliman FNK, Rizk RE, Brake J. Relationship between eggshell porosity eggshell thickness, egg weight loss, and embryonic development in japanese quail eggs. Poultry Science 1994;73:1607-1611.

Too HC, Shibata M, Yayota M, Darras VM, Iwasawa A. Expression of thyroid hormone regulator genes in the yolk sac membrane of the developing chicken embryo. The Journal of reproduction and development, 2017:63:463-472

Tuan RS, Ono T. Regulation of extraembryonic calcium mobilization by the developing chick embryo. Journal of Embryology and Experimental Morphology 1986;97:63-74.

Ulmer-Franco AM, Fasenko GM, O'Dea Christopher EE. Hatching egg characteristics, chick quality, and broiler performance at 2 breeder flock ages and from 3 egg weights. Poultry Science 2010;89:2735-2742.

Willems E, Decuypere E, Buyse J, Everaert N. Importance of albumen during embryonic development in avian species, with emphasis on domestic chicken. World's Poultry Science Journal 2014;70:503-518.

Yuan J, Wang B, Huang Z, Fan Y, Huang C, Hou Z. Comparisons of egg quality traits, egg weight loss and hatchability between striped and normal duck eggs. British Poultry Science 2013;54:265-269.

Zita L, Ledvinka Z, Tumova E, Klesalova L. Technological quality of eggs in relation to the age of laying hens and Japanese quails. Revista Brasileira de Zootecnia 2012;41:2079-2084. 